\title{
Reduced dose iterative reconstruction versus standard dose filtered back projection in detection of bladder tumors
}

\author{
Mohamed Sharafeldeen', Mohamed Shaaban², Asmaa Mahmoud Ramadan², Tarek Rashad ${ }^{2}$ and \\ Mohamed Elsaqa ${ }^{1 *}$ (i)
}

\begin{abstract}
Background: The purpose was to assess radiation dose, image quality, and diagnostic performance of reduceddose scanning with iterative reconstruction (IR) compared with standard-dose with filtered back projection (FBP) with $\mathrm{CT}$ urography for detection of bladder tumor. This study was prospectively conducted on 21 patients with bladder masses. All patients were subjected to two scanning protocols: protocol A (standard dose with FBP) and protocol B (additional limited scan to the pelvis at delayed phase with low dose with IR). Based on body weight ( $<$ or $>80 \mathrm{~kg}$ ), each protocol was subdivided into 2 protocols $A_{1}(130 \mathrm{kVp})$ and $A_{2}(130 \mathrm{kVp})$ and protocols $B_{1}(80 \mathrm{kVp})$ and $B_{2}(110 \mathrm{kVP})$. Radiation dose was assessed in terms of mean $C T$ dose index (CTDI), Dose-length product (DLP) and effective dose (ED). Image quality and diagnostic accuracy were compared in both groups.
\end{abstract}

Results: Mean CTDI, DLP and ED were reduced by average $72.3 \%$ in the $80 \mathrm{kVp}$ protocol $\left(\mathrm{B}_{1}\right)$ and by $36.3 \%$ in 110 $\mathrm{kVp}\left(\mathrm{B}_{2}\right)$ protocol compared to standard-dose protocols. There were significantly lower SNR (signal to noise ratio) between protocol $A_{1}$ and $B_{1}$ at aorta and psoas muscles. Subjective image quality analysis revealed no statistically significant differences between the protocol $A_{2}$ and $B_{2}$ whereas there were significant differences between protocol $A_{1}$ and $B_{1}$ as regards to visual image noise and overall image quality. Diagnostic accuracy was identical among different protocols.

Conclusion: $\mathrm{CT}$ urography with IR scanning showed reduced radiation dose and no difference in detection of urothelial carcinomas from standard dose with FBP despite of degraded image quality in $80 \mathrm{kVp}$ scanning.

Keywords: Reduced dose, CT urography, Bladder mass

\section{Background}

CT Urography (CTU) is one-step technique that allows full evaluation of the urinary tract for urothelial tumors, renal masses and stones in a single examination. Meanwhile, in traditional CTU protocols patients receive radiation dose of about $54 \mathrm{mGy}$, the reduction of radiation dose in CTU has been an important item to be resolved $[1,2]$. Radiation dose reduction with filtered back projection (FBP) reconstruction techniques is associated

\footnotetext{
*Correspondence: mohamed.elsaqa@alexmed.edu.eg

'Department of Urology, Faculty of Medicine, Alexandria University, Sultan Hussein Street, Al Azarita, Alexandria, Egypt

Full list of author information is available at the end of the article
}

with increasing image noises and streak artifacts [3]. With general technical evolution, these drawbacks of FBP have driven the rise of iterative reconstruction (IR) algorithms. The current literature shows that clinical implementation of IR into CT protocols provides essential promise for major improvements in image quality, chiefly noise reduction together with subsequent radiation dose reduction and artifact suppression [3]. The purpose of this study is to compare radiation dose, image quality and diagnostic performance of CTU using reduced-dose scanning with IR versus standard-dose with FBP for detection of urinary bladder tumors. 


\section{Methods}

Twenty-one consecutive patients presented to Alexandria University Hospitals with sonographically diagnosed urinary bladder masses were enrolled in the study between August 2016 and December 2017.

Enrolled patients had serum glomerular filtration rate greater than $60 \mathrm{ml} / \mathrm{min} / 1.73 \mathrm{~m}^{2}$. Patients with contraindications to the use of iodinated contrast media or hip prosthesis were excluded. All patients included in the study were subjected to full history taking including personal data and clinical examination and Routine laboratory investigations.

\section{CT data acquisition}

All patients were subjected to two scanning protocols:

- Protocol A: standard dose with FBP

- Protocol B: additional limited scan to the pelvis at delayed phase with low dose with IR.

\section{Patients groups}

Each protocol is subdivided according to body weight into two protocols:

$A_{1}(130 \mathrm{kVp})$ and $A_{2}(130 \mathrm{kVp})$ and protocols $B_{1}(80$ $\mathrm{kVp})$ and $\mathrm{B}_{2}(110 \mathrm{kVp})$ based on body weight.

Patients with body weight less than $80 \mathrm{~kg}(n=10)$ to standard dose with FBP $130 \mathrm{kVp}$ (protocol $\mathrm{A}_{1}$ ) and additional scan limited to urinary bladder at delayed phase with low dose with IR $80 \mathrm{kVp}$ (protocol $\mathrm{B}_{1}$ ) while patients with more than $80 \mathrm{~kg}(n=11)$ into standard dose $130 \mathrm{kvp}$ (protocol $\mathrm{A}_{2}$ ) and additional scan limited to urinary bladder at delayed phase with (protocol $\mathrm{B}_{2}$ ); low dose $110 \mathrm{kvp}$.

All CT examinations were performed with SOMATOM Perspective 64 MDCT (Siemens Healthcare, Forchheim, Germany) at Alexandria Main University Hospital.

Image acquisition parameters were detector collimation 1.2 , slice thickness $1.5 \mathrm{~mm}$, field of view $51 \mathrm{~cm}$, rotation time $0.6 \mathrm{~s}$, helical pitch 0.6 , tube current was automatically modulated by the machine, and ranged from 165 to 200 mAs. Additional scan limited to urinary bladder was acquired at delayed phase with scan parameters identical to that of standard dose except for the tube voltage which was reduced to $80 \mathrm{kVp}$ (protocol $\mathrm{B}_{1}$ ) or $110 \mathrm{kVp}$ (protocol $\mathrm{B}_{2}$ ) according to body weight.

The injection protocols were the same in all examination, $100 \mathrm{ml}$ of iohexol (Omnipaque $300 \mathrm{mg} / \mathrm{ml}$; GE Healthcare Ireland, Cork, Ireland) which was administered into antecubital vein with flow rate of $2-4 \mathrm{ml} / \mathrm{s}$ via a pump injector (Medrad mark V, Bayer healthcare, Pennsylvania, USA). Patients consumed $600 \mathrm{ml}$ water orally while waiting for 1 hour before undergoing CT to allow distention of the urinary bladder. Images were obtained at unenhanced, nephrographic (90-110 s after administration of IV contrast medium), and delayed (420 s after administration of contrast medium) phases for standard FBP protocols, scan range started from above the dome of diaphragm to ischial bones as determined from the AP topogram. Delayed phase only for low-dose protocols limited to the urinary bladder as determined from the prior scans.

\section{Image reconstruction}

Raw data of reduced-dose CT examinations in the study group were reconstructed using both mathematical algorithms, FBP and IR (SAFIRE). SAFIRE has 5 preset strength levels. According to recommendations by the vendor, a medium strength level of 3 was applied to all reconstructions in our study. Reconstruction parameters included a B20s + smooth convolution kernel for FBP and the equivalent I30/3+ medium smooth kernel for IR.

\section{Radiation dose report (for the pelvis)}

For each patient, volume CT dose index (CTDI) and the dose-length product (DLP) were recorded from the automatically generated patient protocol. The effective radiation dose was then estimated in millisieverts $(\mathrm{mSv})$ (the dose-length product multiplied by the conversion factor of 0.015) [4] for each scan.

\section{Image analysis}

Both objective and subjective image quality were assessed for all patients in a blinded and randomized fashion using Syngo via Workplace; Siemens Healthcare.

\section{Objective image quality}

The CT attenuation value (in Hounsfield units HU) of aorta and psoas muscle, image noise (standard deviation of CT attenuation values SD), signal-to-noise ratio (SNR), and contrast-to-noise ratio (CNR) were assessed within the identical two areas (psoas muscle and abdominal aorta) by using a region of interest (ROI) of at least $40 \mathrm{~mm}^{2}$ at level of L4 before bifurcation of abdominal aorta in delayed phases by one radiologist with 5 years of experience.

SNR and CNR were calculated based on following standard equations, according to Bhosale P., et al. [5]:

$$
\begin{aligned}
& \mathrm{SNR}=\frac{\text { mean HU of tissue in ROI }}{\mathrm{SD} \text { of tissue in ROI }} \\
& \mathrm{CNR}=\frac{\text { mean HU of tissue in ROI-mean HU of background fat }}{\mathrm{SD} \text { of background fat }}
\end{aligned}
$$

\section{Subjective image quality}

Subjective image quality and bladder carcinoma were initially assessed independently by two experienced radiologists with more than 20 and 12 years of experience in abdomino-pelvic CT performed visual assessments in blinded randomized fashion. The radiologists rated 
Table 1 Distribution of the studied cases according to demographic data ( $n=21)$

\begin{tabular}{llll}
\hline & $\begin{array}{l}\text { Total } \\
(n=21)\end{array}$ & $\begin{array}{l}\text { Protocols according to weight } \\
\text { Protocol A1, B1 } 380 \mathrm{~kg} \\
(n=10)\end{array}$ & $\begin{array}{l}\text { Protocol A1, B2 }>80 \mathrm{~kg} \\
(n=11)\end{array}$ \\
\hline $\begin{array}{lll}\text { Sex (\%) } \\
\text { Male }\end{array}$ & $18(85.7)$ & $8(80)$ & $10(90.9)$ \\
$\quad \begin{array}{l}\text { Female } \\
\text { Age (years) }\end{array}$ & $3(14.2)$ & $2(20)$ & $1(9.09)$ \\
Min.-max & & $55-70$ & $50-71$ \\
Mean \pm SD & $50-71$ & $61.4 \pm 4.9$ & $63.6 \pm 6.15$ \\
Weight (kg) & $62.5 \pm 5.52$ & & $85-110$ \\
Min.-max. & & $64-78.0$ & $92.4 \pm 7.26$ \\
Mean \pm SD & $64.0-110.0$ & $71.0 \pm 5.24$ & \\
\hline
\end{tabular}

visual image noise and according to a three-point score (1, unacceptable; 2 , average; 3 , minimal), and overall image quality according to a 3 -point score (1, unacceptable; 2, good; 3 , excellent). The presence or absence of bladder lesion was assessed (0, absent; 1 , present). Differences between two readers were solved by consensus. Results were then compared with cystoscopic examination results and pathological analysis.

\section{Statistical analysis}

Statistical analysis was performed with SPSS 20.0 (IBM Corp. Released 2013. IBM SPSS Statistics for Windows, Version 22.0. Armonk, NY: IBM Corp.).

\section{Results}

\section{Demographic patient characteristics}

Detailed characteristics of patients were provided in Table 1. The 21 patients underwent standard-dose CT urography protocol A and additional limited scan to the pelvis at delayed phase protocol B. scan protocols were subdivided into 4 groups protocol $\mathrm{A}_{1}$ and $\mathrm{B}_{1}$ (patients $\leq$
$80 \mathrm{~kg} ; 10$ patients $47.6 \%$ ) and protocol $A_{2}$ and $B_{2}$ (patients > $80 \mathrm{~kg} ; 11$ patients $52.3 \%$ ).

\section{Radiation dose}

The mean volume CT dose indices (CTDI) for group $\mathrm{A} 1(130 \mathrm{kVp})$ and $\mathrm{B} 1(80 \mathrm{kVp})$ were $6.64 \pm .40$ and $1.95 \pm$ $1.12 \mathrm{mGy}$, respectively, while for groups A2(130 kVp) and $\mathrm{B} 2(110 \mathrm{kVp})$, it was $6.71 \pm .46$ and $4.58 \pm .35 \mathrm{mGy}$, respectively; this represents decrease by $71.5 \%$ for 80 $\mathrm{kVp}$ protocol (B1) and $31.7 \%$ for the $110 \mathrm{kVp}$ protocol (B2) versus standard-dose protocols; this is described in details in the Table 2.

The mean DLP in protocol A1(130 kVp) was 323.91 mGy-cm \pm 25.16 and for protocol B1 (80 kVp) 97.43 $\mathrm{mGy}-\mathrm{cm} \pm 54.25$ which is $74.7 \%$ decrease and for protocol A2 $(130 \mathrm{kVp}) 339.78 \mathrm{mGy}-\mathrm{cm} \pm 25.70$ and for protocol B2(110 kVp) $213.31 \mathrm{mGy}-\mathrm{cm} \pm 38.52$ which is $39.1 \%$ decrease as shown in Table 2. The mean ED for protocol $\mathrm{A} 1(130 \mathrm{kVp})$ was $5.49 \mathrm{mSv} \pm .43$ and for protocol B1 (80 $\mathrm{kVp}$ )was $1.65 \pm .92$ with $70.8 \%$ decrease while for protocol A2 $(130 \mathrm{kVp})$ and B2 $(110 \mathrm{kVp})$ mean ED was 5.77

Table 2 Descriptive analysis of the studied cases according to CTDI in mGy, DLP in mGy-cm and ED in mSv

\begin{tabular}{|c|c|c|c|c|c|c|}
\hline & \multicolumn{2}{|c|}{ Weight $\leq 80 \mathrm{~kg}(n=10)$} & \multicolumn{2}{|c|}{ Weight $>80 \mathrm{~kg}(n=11)$} & \multirow{2}{*}{$\begin{array}{l}P \text { value } \\
\text { (A1 vs. } \\
\text { B1) }\end{array}$} & \multirow{2}{*}{$\begin{array}{l}P \text { value } \\
\left(A_{2} \text { vs. }\right. \\
\left.B_{2}\right)\end{array}$} \\
\hline & $\begin{array}{l}\text { Standard dose } \\
\text { Protocol } A_{1}\end{array}$ & 80 kVp Protocol B1 & $\begin{array}{l}\text { Standard dose } \\
\text { Protocol A2 }\end{array}$ & $\begin{array}{l}110 \mathrm{kVp} \\
\text { Protocol B2 }\end{array}$ & & \\
\hline \multicolumn{7}{|l|}{ CTDI in mGy } \\
\hline Min.-max. & $6.40-7.34$ & $1.25-4.914$ & $6.42-7.46$ & $4.16-4.93$ & $\left(0.002^{*}\right)$ & $\left(0.002^{*}\right)$ \\
\hline Mean $\pm S D$ & $6.64 \pm .40$ & $1.95 \pm 1.12$ & $6.71 \pm .46$ & $4.58 \pm .35$ & & \\
\hline \multicolumn{7}{|c|}{ DLP in mGy (cm) } \\
\hline Min.-Max. & $279.8-357.84$ & $63.05-240.17$ & 298.12-378.17 & $142.01-253.72$ & $\left(0.002^{*}\right)$ & $\left(0.002^{*}\right)$ \\
\hline Mean \pm SD & $323.91 \pm 25.16$ & $97.43 \pm 54,25$ & $339.78 \pm 25.70$ & $213.31 \pm 38.52$ & & \\
\hline \multicolumn{7}{|l|}{$\mathrm{ED}$ in $\mathrm{mSv}$} \\
\hline Min-max & $4.75-6.08$ & $1.07-4.08$ & $5.06-6.42$ & $2.41-4.31$ & $\left(0.002^{*}\right)$ & $\left(0.002^{*}\right)$ \\
\hline Mean \pm SD & $5.49 \pm .431$ & $1.65 \pm .92$ & $5.77 \pm .43$ & $3.62 \pm .65$ & & \\
\hline
\end{tabular}

$P$ values for Wilcoxon signed ranks test for comparing between standard FBP and low dose with IR

*Statisticallysignificant at $P \leq 0.05$ 


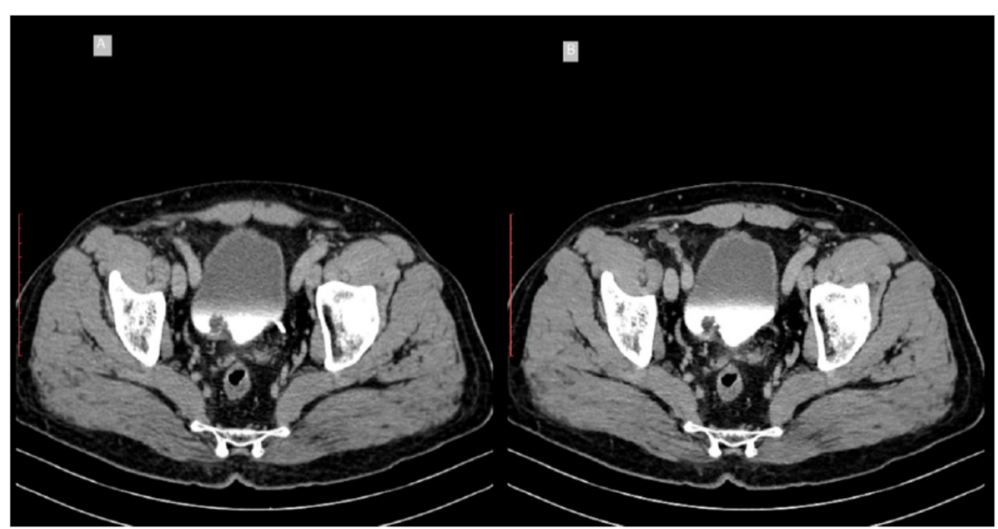

Fig. 1 Male patient, 68 years old, $98 \mathrm{~kg}$. a Axial CT urogram obtained during excretory phase demonstrate CT urography standard dose with FBP protocol A2, SNR were 4.25 and 2.88 at aorta and psoas muscle, respectively. CNR were 11.68 and 10.45 at aorta and psoas respectively. b Axial CT urogram with low dose with IR protocol B2, SNR were 4.55 and 2.91 at aorta and psoas respectively. CNR were 13.46 and 12.02 at aorta and psoas, respectively. Both protocols showing bladder mass

$\mathrm{mSv} \pm .43$ and $3.62 \mathrm{mSv} \pm .65$, respectively, with $38.1 \%$ decrease (Fig. 1).

\section{Objective image quality assessment SNR values}

- Protocol $A_{1}$ vs. Protocol $B_{1}$ : There were significant differences in SNRs between protocol $A_{1}$ and $B_{1}$ at aorta and psoas muscles.

- Protocol A2 vs. Protocol B2: No significant differences were seen between protocol $\mathrm{A}_{2}$ and $\mathrm{B}_{2}(P$ $\leq 0.05$ for all). The results of the objective image analyses are listed in Table 3.

\section{CNR values}

There was no significant difference at CNR between different groups, as listed in Table 3.

\section{Subjective image quality assessment}

Protocol $A_{1}$ vs. Protocol $B_{1}$ : There were significant differences between protocol $A_{1}(130 \mathrm{kVp})$ and $B_{1}(80 \mathrm{kVp})$ as regards to visual image noise, and overall image quality as listed in (Table 3).

Protocol $\mathrm{A}_{2}$ vs. Protocol $\mathrm{B}_{2}$ : There were no significant differences between protocol $A_{2}(130 \mathrm{kVp})$ and $B_{2}(110$ $\mathrm{kVp}$ ) as shown in Table 3.

Table 3 Descriptive analysis of the studied cases according to mean objective image quality and subjective image quality

\begin{tabular}{|c|c|c|c|c|c|c|c|c|}
\hline & & & \multicolumn{2}{|c|}{ Weight $\leq 80 \mathrm{~kg}(n=10)$} & \multicolumn{2}{|c|}{ Weight > 80 kg $(n=11)$} & \multirow{2}{*}{$\begin{array}{l}P \text { value } \\
\text { for } A_{1} \text { vs. } B_{1}\end{array}$} & \multirow{2}{*}{$\begin{array}{l}P \text { value } \\
\text { for } \\
A_{2} \text { vs. } B_{2}\end{array}$} \\
\hline & & & $\begin{array}{l}\text { Standard dose } \\
\text { Protocol } A_{1}\end{array}$ & $\begin{array}{l}80 \mathrm{kVp} \\
\text { Protocol } \mathrm{B}_{1}\end{array}$ & $\begin{array}{l}\text { Standard dose } \\
\text { Protocol } A_{2}\end{array}$ & $\begin{array}{l}110 \mathrm{kVp} \\
\text { Protocol } \mathrm{B}_{2}\end{array}$ & & \\
\hline \multirow{12}{*}{$\begin{array}{l}\text { Objective image } \\
\text { quality }\end{array}$} & Mean SNR & Aorta & & & & & & \\
\hline & & Min-max. & $2.38-7.30$ & $1.45-5.66$ & $1.78-8.71$ & $2.14-9.39$ & $\left(0.007^{*}\right)$ & $(0.082)$ \\
\hline & & Mean \pm SD & $5.10 \pm 1.64$ & $3.48 \pm 1.56$ & $5.24 \pm 2.17$ & $5.14 \pm 2.24$ & & \\
\hline & & Psoas Ms & & & & & & \\
\hline & & Min.-max. & $2.07-4.56$ & $1.51-3.02$ & $1.21-9.71$ & $1.39-4.08$ & $\left(0.006^{*}\right)$ & $(0.82)$ \\
\hline & & Mean \pm SD & $3.72 \pm 0.84$ & $2.23 \pm 0.49$ & $3.75 \pm 2.32$ & $2.94 \pm 0.86$ & & \\
\hline & Mean CNR & Aorta & & & & & & \\
\hline & & Min.-max. & $8.42-22.72$ & $6.64-18.63$ & $5.64-20.29$ & $9.1-29.35$ & $(0.085)$ & $(0.29)$ \\
\hline & & Mean \pm SD & $13.94 \pm 4.55$ & $10.24 \pm 3.75$ & $13.39 \pm 4.09$ & $15.50 \pm 6.9$ & & \\
\hline & & Psoas Ms & & & & & & \\
\hline & & Min.-max. & $7.15-17.96$ & $5.47-16.39$ & $4.98-21.63$ & $7.88-23.42$ & $(0.42)$ & $(0.34)$ \\
\hline & & Mean \pm SD & $12.03 \pm 3.55$ & $8.25 \pm 2.16$ & $12.02 \pm 4.53$ & $13.04 \pm 5.07$ & & \\
\hline \multirow{2}{*}{$\begin{array}{l}\text { Subjective image } \\
\text { quality }\end{array}$} & Image noise & & $3(1-3)$ & $2(1-3)$ & $3(2-3)$ & $3(2-3)$ & $\left(0.001^{*}\right)$ & 1 \\
\hline & Image quality & & $3(1-3)$ & $2(1-3)$ & $3(2-3)$ & $3(2-3)$ & $(0.001 *)$ & $(0.546)$ \\
\hline
\end{tabular}

$P$ values for Wilcoxon signed ranks test for comparing between standard FBP and low dose with IR

* Statistically significant at $P \leq 0.05$ 


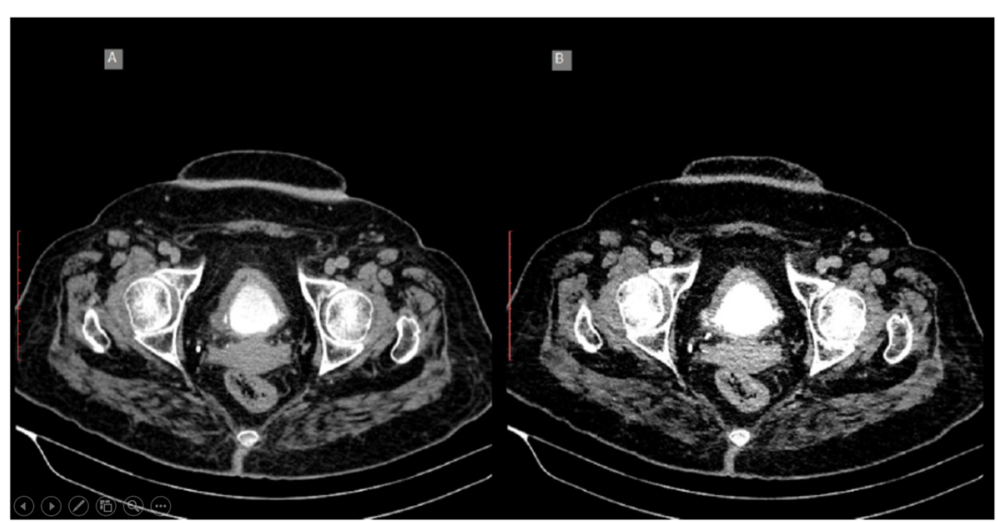

Fig. 2 Female patient, 63 years old,70 kg. a Axial CT urography with standard dose CT protocol A1, SNR were 4.79 and 3.08 at aorta and psoas muscle respectively and CNR were 9.4 and 8.3 at aorta and psoas respectively. b Axial CT urography with low dose with IR protocol B1, SNR were 2.26 and 2.07 and CNR were 6.6 and 5.6 at aorta and psoas, respectively. Both protocols showing diffuse mural thickening which proved to be TCC

\section{Detection of bladder carcinoma}

Diagnosis was identical among different protocols as regards per patient-level analyses among observers. Diagnostic accuracy of CT urography at standard and low-dose protocols was 91.3\% (Fig. 2). Detailed results are listed in Table 4 . Out of the 21 patients, 19 patients proved to have urothelial bladder cancer (90.4\%); 1 of them had urothelial carcinoma with glandular and squamous cell differentiation which is considered bad prognostic factor. One patient had small cell carcinoma (4.7\%) and One patient proved to be negative (chronic cystitis) as reported in CTU and proved by cystoscopy and biopsy result.

Two cases having multiple superficial small papillary lesions were reported as normal in both standard- and lowdose protocols CT while in cystoscopy, lesions were noted and biopsy results revealed urothelial bladder carcinomas. Per lesion diagnosis was the same for standard-dose and low-dose protocols. Diagnostic accuracy per lesion at standard-dose and low-dose protocols were $78.8 \%$. Superficial small papillary lesions were missed in various cases at CT urography. Detailed results are listed in Table 4.

\section{Discussion}

The radiation dose of CT urography is estimated to be approximately 15 to $20 \mathrm{mSv}$ according to the ESUR guideline. The increasing dose awareness in the radiological and the general medical community has driven radiologists as well as equipment manufacturers to optimize CT imaging procedures with the aim of dose reduction [6].

In our study, we compare between standard dose CT with FBP and low-dose CT with IR at delayed phase only. By applying reduced-dose protocols in our study for detection of bladder cancer, CTDI and DLP and ED was reduced by average $72.3 \%$ in the $80 \mathrm{kVp}$ protocol $\left(\mathrm{B}_{1}\right)$ and by $36.3 \%$ in $110 \mathrm{kVp}\left(\mathrm{B}_{2}\right)$ protocol in comparison with standard-dose protocols, protocol $A_{1}$ and protocol $A_{2}$. There is significant decrease in radiation dose, the same as reported in previous studies [7-10].

In our study, attenuation and noise of tissues of interest were increased significantly when the tube voltage setting was lowered to $80 \mathrm{kVp}$, which resulted in lower SNR with $3.48 \pm 1.56$ at aorta and $2.23 \pm 0.49$ at psoas

Table 4 Diagnostic performance per patient and per lesion in detection of bladder cancer

\begin{tabular}{|c|c|c|c|c|c|}
\hline & & $\begin{array}{l}\text { Standard dose } \\
\text { with FBP protocols }\end{array}$ & 95\% Confidence interval & $\begin{array}{l}\text { Low dose with } \\
\text { IR protocols }\end{array}$ & $\begin{array}{l}95 \% \text { confidence } \\
\text { interval }\end{array}$ \\
\hline \multirow[t]{4}{*}{ Detection per patient } & Sensitivity & $90.91 \%$ & $70.84-98.88 \%$ & $90.91 \%$ & $70.84-98.88 \%$ \\
\hline & Positive predictive value & $100 \%$ & - & $100 \%$ & - \\
\hline & Negative predictive value & $33.33 \%$ & $11.77-65.21 \%$ & $33.33 \%$ & $11.77-65.21 \%$ \\
\hline & Accuracy & $91.30 \%$ & $71.96-98.93 \%$ & $91.30 \%$ & $71.96-98.93 \%$ \\
\hline \multirow[t]{4}{*}{ Detection per lesion } & Sensitivity & $78.26 \%$ & $63.64-89.05 \%$ & $78.26 \%$ & $63.64-89.05 \%$ \\
\hline & Positive predictive value & $100 \%$ & - & $100 \%$ & - \\
\hline & Negative predictive value & $9.09 \%$ & $5.46-14.75 \%$ & $9.09 \%$ & $5.46-14.75 \%$ \\
\hline & Accuracy & $78.72 \%$ & $64.34-89.30 \%$ & $78.72 \%$ & $64.34-89.30 \%$ \\
\hline
\end{tabular}


muscle in comparison with standard-dose protocol where SNR was $5.10 \pm 1.64$ at aorta and $3.72 \pm 0.84$ at psoas muscle. While in previous studies [7, 9], SNR of low-dose protocols were higher compared to standarddose protocols. Difference between studies at noise levels and SNR at $80 \mathrm{kVp}$ protocol could be attributed to variability in tube voltage and tube current. While there was no significant difference at CNR between standard-dose protocol $\left(\mathrm{A}_{1}\right)$ and $80 \mathrm{kVp}$ protocol $\left(\mathrm{B}_{1}\right)$.

As regards to subjective image assessment, increased visual image noise degraded the image quality in $80 \mathrm{kVp}$, but did not affect the diagnostic accuracy, same as in Bahn et al. [7].

Whereas in $110 \mathrm{kVp}$, there were no significant differences were noted at SNR and CNR in comparison with standard-dose protocol $A_{2}$. Subjective image analysis was similar to standard dose, for image noise and overall image quality. $B_{2}$ protocol showed better lesion conspicuity and even a less image noise and better image quality than standard dose at $18.8 \%$ of cases for protocol $B_{2}$ as reported by two experienced radiologists. This is consistent with Gervise et al. [9] and Bahn et al. [7] which confirmed that it is possible to perform an acquisition with $100 \mathrm{kV}$ for most patients including overweight patients and obese patients.

Our results showed that the detection of urothelial carcinomas by the reduced-dose images with IR was the same as standard-dose with FBP. Lesion and patientbased accuracies were $78.72 \%$ and $91.30 \%$, respectively, among different protocols that had the same range of patient body weight. As reported with Juri et al. [4] and Bahn et al. [7]. The higher accuracies may be attributed to the larger tumor size. Superficial low-grade tumors are still challenging to detect regardless of standard-dose images with FBP or reduced-dose images with IR.

\section{Limitations of the study}

This includes small number of patients in this study. A study with larger number of patients is needed. Also, the study only assessed bladder carcinomas, and did not asses other related disease entities, including upper urinary tract abnormalities or lymphadenopathy. Moreover, we did not compare between different CT vendors.

\section{Conclusion}

This study showed the use of reduced-dose IR images was associated with significant reduction in radiation dose with no difference at diagnostic detection of bladder carcinomas compared with the use of standard dose FBP images, though IR was associated with an increased noise in $80 \mathrm{kVp}$ images. These results may have important clinical implications using a low-tube voltage scan to reduce cumulative radiation exposure without compromising lesion detection.

\section{Abbreviations}

CT: Computed tomography; CTDI: CT dose index; CTU: Computed tomography urography; CNR: Contrast-to-noise ratio; DLP: Dose-length product; ED: Effective dose; FBP: Filtered back projection; HU: Hounsfield units; IR: Iterative Reconstruction; KV: Kilovoltage; KVp: Kilovoltage peak; MDCT: Multidetector computed tomography; mAs: Milliampere-seconds; mGy: Megagray; ROI: Region of interest; SD: Standard deviation; SNR: Signalto-noise ratio

\section{Acknowledgements \\ None.}

\section{Authors' contributions}

MS: study design, recruitment of cases, supervision, revision). MSS: idea of research, coordination between investigators. AMR: writing, collection of data, statistics). TR supervising, revision. ME: contribution to data collection, contribution to writing. All authors have read and approved the manuscript.

\section{Funding}

None.

\section{Availability of data and materials}

The datasets used and/or analyzed during the current study are available from the corresponding author on reasonable request.

Ethics approval and consent to participate

- Done and the study was submitted to and approved by our ethics committee of Faculty of Medicine, Alexandria University.

- Ethics committee's reference number: not available

- Formal written consent was taken from all participants

Consent for publication

Consent was taken from all participants.

\section{Competing interests}

The authors declare that they have no competing interests.

\section{Author details}

${ }^{1}$ Department of Urology, Faculty of Medicine, Alexandria University, Sultan Hussein Street, Al Azarita, Alexandria, Egypt. ²Department of Radiology, Faculty of Medicine, Alexandria University, Alexandria, Egypt.

Received: 22 April 2020 Accepted: 27 May 2020

Published online: 15 June 2020

References

1. Nawfel RD, Judy PF, Schleipman AR et al (2004) Patient radiation dose at CT urography and conventional urography. Radiology 232(1):126-132

2. Eikefjord EN, Thorsen F, Rørvik J (2007) Comparison of effective radiation doses in patients undergoing unenhanced MDCT and excretory urography for acute flank pain. Am J Roentgenol 188(4):934-939

3. Geyer LL, Schoepf UJ, Meinel FG et al (2015) State of the art: iterative CT reconstruction techniques. Radiology 276(2):339-357

4. Juri H, Matsuki M, Inada Y et al (2013) Low-dose computed tomographic urography using adaptive iterative dose reduction 3-dimensional: comparison with routine-dose computed tomography with filtered back projection. J Comput Assist Tomogr 37(3):426-431

5. Bhosale P (2015) Comparing CNR, SNR, and Image Quality of CT Images Reconstructed with Soft Kernel, Standard Kernel, and Standard Kernel plus ASIR 30\% Techniques. Int J Radiol 2(2):60-65

6. Von Falck C, Bratanova V, Rodt et al (2013) Influence of sinogram affirmed iterative reconstruction of $\mathrm{CT}$ data on image noise characteristics and lowcontrast detectability: an objective approach. PLoS One 8(2):e56875

7. Bahn YE, Kim SH, Kim MJ et al (2015) Detection of urothelial carcinoma: Comparison of reduced-dose iterative reconstruction with standard-dose filtered back projection. Radiology 279(2):471-480

8. Funama Y, Awai K, Nakayama Y et al (2005) Radiation dose reduction without degradation of low-contrast detectability at abdominal multisection CT with a low-tube voltage technique: phantom study. Radiology 237(3): 905-910 
9. Gervaise A, Naulet P, Beuret F et al (2014) Low-dose CT with automatic tube current modulation, adaptive statistical iterative reconstruction, and low tube voltage for the diagnosis of renal colic: impact of body mass index. Am J Roentgenol 202(3):553-560

10. Marin D, Nelson RC, Schindera ST et al (2010) Low-Tube-Voltage, High-TubeCurrent Multidetector Abdominal CT: Improved Image Quality and

Decreased Radiation Dose with Adaptive Statistical Iterative Reconstruction Algorithm—Initial Clinical Experience. Radiology 254(1):145-153

\section{Publisher's Note}

Springer Nature remains neutral with regard to jurisdictional claims in published maps and institutional affiliations.

\section{Submit your manuscript to a SpringerOpen ${ }^{\circ}$ journal and benefit from:}

- Convenient online submission

- Rigorous peer review

- Open access: articles freely available online

High visibility within the field

- Retaining the copyright to your article

Submit your next manuscript at $\boldsymbol{\nabla}$ springeropen.com 